\title{
Ultrasound wave propagation through rough interfaces: Iterative methods
}

\author{
Arthur P. Berkhoff and Johan M. Thijssen \\ Biophysics Laboratory, Department of Ophthalmology, Nijmegen University Hospital, P.O. Box 9101, \\ 6500 HB Nijmegen, The Netherlands \\ Peter M. van den Berg \\ Laboratory of Electromagnetic Research, Faculty of Electrical Engineering, Centre of Technical \\ Geoscience, Delft University of Technology, P.O. Box 5031, 2600 GA Delft, The Netherlands
}

(Received 8 May 1995; revised 15 September 1995; accepted 20 November 1995)

\begin{abstract}
Two iterative methods for the calculation of acoustic transmission through a rough interface between two media are compared. The methods employ a continuous version of the conjugate gradient technique. One method is based on plane-wave expansions and the other on boundary integral equations and Green's functions. A preconditioner is presented which improves the convergence for spectra that include evanescent modes. The methods are compared with regard to computational efficiency, rate of convergence, and residual error. The sound field differences are determined for a focused ultrasound beam distorted by surfaces having a Gaussian roughness spectrum. The differences are evaluated from the root-mean-square differences on the rough surface and in the focal plane. (C) 1996 Acoustical Society of America.
\end{abstract}

PACS numbers: 43.20.Fn, 43.30.Hw, 43.80.Qf

\section{INTRODUCTION}

In echographic imaging with large array transducers, wave distortions in intervening tissue layers often are the major cause for image distortions. Various correction methods have been proposed to obtain better images for these cases. ${ }^{1-4}$ In order to test the applicability and limits of the correction methods, it is important to have an accurate and efficient computational model describing the propagation through these distorting tissue layers. The sound propagation through tissue is often modeled as the propagation through a layer consisting of a cascade of thin sublayers, where each sublayer acts upon the incident wave as a random time-shift operator. ${ }^{5}$ Various papers, ${ }^{5-7}$ however, report that the wave modification cannot be described sufficiently with these models and that refraction effects also should be taken into account. In particular, ultrasound propagation through female breast $^{5,7}$ suffers from severe refractive errors. In abdominal animal tissue ${ }^{6}$ strong multipath components were noticed, with refraction as a possible cause. Recently, therefore, we have tried to come up with an efficient numerical method ${ }^{9}$ to model the process of refraction, which is attacked by solving the numerical problem of wave propagation through an irregular interface between two uniform media. Of course, the present model should be extended with distributed wave aberrations to get a more complete description of wave propagation through human tissue.

If the medium parameters within each layer are assumed to be constant, then the computational problem can be reduced to the problem of finding the acoustic variables on the interfaces of the layered configuration. The discretization of the problem leads to a large number of unknowns. Due to the size of the numerical problem, iterative methods are essential. Iterative methods can lead to dramatically reduced storage requirements and total computation time, especially for
3-D calculations. Also for large 2-D problems the requirements on computation time and memory resources can be prohibitive.

In this paper, two iterative methods are described for the calculation of reflection and transmission at a rough interface between two media. Both methods are based on a continuous version of the conjugate gradient technique. ${ }^{8}$ One method is based on plane-wave expansions ${ }^{9}$ while the other method is based on boundary integral equations and free-space Green's functions. ${ }^{10}$ Although the application deals with pulse-echo ultrasound, the domain of analysis is the frequency domain. An analysis in the frequency domain has the advantage that the strongly frequency-dependent absorption and dispersive sound speed can be incorporated quite easily. Time-domain results are obtained by analyzing the problem at several frequencies and subsequently calculating the inverse (temporal) Fourier transform. Wave propagation through random interfaces can be analyzed by evaluating a large number of interfaces. ${ }^{11}$ According to Altmeyer, ${ }^{12}$ typical acoustic interfaces in human tissue have a root-mean-square surface height of at least $0.5 \mathrm{~mm}$. Characteristic length scales of these surfaces are not given by Altmeyer. The soft tissues show low contrast between the different tissue layers, with a difference in sound speed typically lying between zero and five percent.

The extensions and novel techniques presented in this paper are the preconditioning scheme for the iterative planewave method of Ref. 9, an efficient preconditioner for this scheme, the comparison of the iterative methods, and the application of rigorous computational techniques to propagation through refractive rough interfaces similar to those occurring in human skin and subcutaneous layers.

\section{FORMULATION OF THE PROBLEM}

The rough interface is assumed to be a local deformation of an otherwise plane boundary at $z=0$, where a point in 


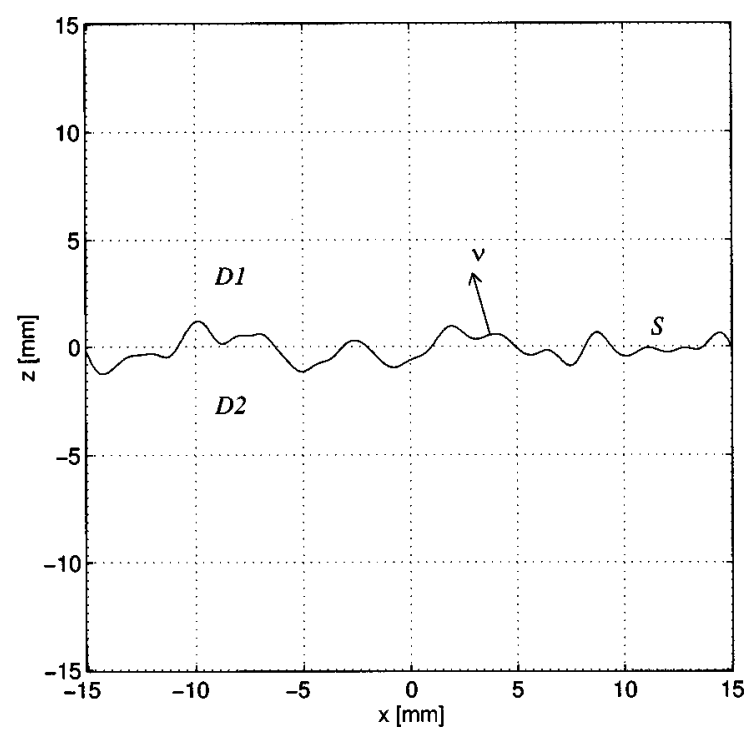

FIG. 1. Configuration and a realization of a rough surface having a Gaussian roughness spectrum with a correlation length $L=1 \mathrm{~mm}$ and a rms surface height $h=0.5 \mathrm{~mm}$.

space is specified by its orthogonal coordinates $\mathbf{x}=(x, y, z)$. A 2-D configuration is shown in Fig. 1. The analysis is carried out in the temporal frequency domain with angular frequency $\omega$ where the complex time factor $e^{-i \omega t}$ is suppressed. The two fluidlike media occupy the domains $\mathscr{D}_{1}$ and $\mathscr{D}_{2}$, respectively, and are assumed to be linear, homogeneous, and isotropic with respective mass densities $\rho_{1}$ and $\rho_{2}$ and compressibilities $\kappa_{1}$ and $\kappa_{2}$. Furthermore, both media exhibit some losses and the real and imaginary parts of $\rho$ and $\kappa$ satisfy the Kramers-Kronig causality relations. The interface is denoted by $\mathscr{S}$. Pressure and the particle velocity variables are denoted by $P$ and $\mathbf{V}$, respectively. In $\mathscr{D}_{1}$, a source of finite extent generates a wave incident upon $\mathscr{S}$. The incident wave is denoted by $\left\{P_{i}, \mathbf{V}_{i}\right\}$. The total field in $\mathscr{D}_{1}$ is written as the superposition of the incident field and the reflected field $\left\{P_{r}, \mathbf{V}_{r}\right\}$. The total field in $\mathscr{D}_{2}$ is the transmitted field $\left\{P_{t}, \mathbf{V}_{t}\right\}$. In two subsequent sections, one direct and two iterative methods are described for the numerical solution of the problem.

\section{INTEGRAL EQUATION METHODS}

\section{A. Direct solution method}

Assuming that the contribution from the parts of the contour integrals at infinity vanish, leads, at the interface $\mathscr{S}$, to the simultaneous integral equations ${ }^{10}$

$$
\begin{aligned}
& \frac{1}{2} P(\mathbf{x})-\int_{\mathbf{x}^{\prime} \in \mathscr{S}}\left[\Gamma_{1}\left(\mathbf{x} \mid \mathbf{x}^{\prime}\right) \mathbf{V}\left(\mathbf{x}^{\prime}\right)+\boldsymbol{\Lambda}_{1}\left(\mathbf{x} \mid \mathbf{x}^{\prime}\right) P\left(\mathbf{x}^{\prime}\right)\right] \\
& \cdot \boldsymbol{\nu}\left(\mathbf{x}^{\prime}\right) d \mathbf{x}^{\prime}=P_{i}(\mathbf{x}), \quad \mathbf{x} \in \mathscr{S}, \\
& \frac{1}{2} P(\mathbf{x})+\int_{\mathbf{x}^{\prime} \in \mathscr{S}}\left[\Gamma_{2}\left(\mathbf{x} \mid \mathbf{x}^{\prime}\right) \mathbf{V}\left(\mathbf{x}^{\prime}\right)+\boldsymbol{\Lambda}_{2}\left(\mathbf{x} \mid \mathbf{x}^{\prime}\right)\right] \cdot \boldsymbol{\nu}\left(\mathbf{x}^{\prime}\right) d \mathbf{x}^{\prime} \\
& \quad=0, \quad \mathbf{x} \in \mathscr{S},
\end{aligned}
$$

where

$$
\begin{aligned}
& \Gamma_{1,2}\left(\mathbf{x} \mid \mathbf{x}^{\prime}\right)=-i \rho \omega G_{1,2}\left(\mathbf{x} \mid \mathbf{x}^{\prime}\right), \\
& \Lambda_{1,2}\left(\mathbf{x} \mid \mathbf{x}^{\prime}\right)=-\nabla_{\mathbf{x}} G_{1,2}\left(\mathbf{x} \mid \mathbf{x}^{\prime}\right),
\end{aligned}
$$

$G_{1,2}\left(\mathbf{x} \mid \mathbf{x}^{\prime}\right)$ is the volume injection Green's function, and $\nabla_{\mathbf{x}}$ the spatial derivative at point $\mathbf{x}$. For $2 \mathrm{D}$ we have $G_{1,2}\left(\mathbf{x} \mid \mathbf{x}^{\prime}\right)$ $=i H_{0}^{(1)}\left(k_{1,2}\left|\mathbf{x}-\mathbf{x}^{\prime}\right|\right) / 4$, with $H_{0}^{(1)}$ the zero-order Hankel function representing outgoing waves and $k_{1}=\omega\left(\rho_{1} \kappa_{1}\right)^{1 / 2}$ and $k_{2}=\omega\left(\rho_{2} \kappa_{2}\right)^{1 / 2}$ the (complex) wave numbers in $\mathscr{D}_{1}$ and $\mathscr{D}_{2}$, respectively.

The integral equations relate the pressure $P$ at a point $\mathbf{x}$ on the interface $\mathscr{S}$ to surface integrals along $\mathscr{S}$ involving pressure and the normal component of the particle velocity. The outward normal of the surface $\mathscr{S}$ at $\mathbf{x}$, pointing into $\mathscr{D}_{1}$, is denoted by $\boldsymbol{\nu}(\mathbf{x})$. Equations (1) and (2) constitute a system of two integral equations with two unknown quantities, viz., $P(x)$ and $\boldsymbol{\nu}(\mathbf{x}) \cdot \mathbf{V}(\mathbf{x})$ on $\mathscr{S}$. It is noted that the integrals in the left-hand sides of (1) and (2) have to be interpreted as their principle values, i.e., the integrals are, when necessary, calculated by a limiting procedure that excludes the singularity at $\mathbf{x}=\mathbf{x}^{\prime}$ in a symmetrical manner. Once the solution to $P(\mathbf{x})$ and $\boldsymbol{\nu}(\mathbf{x}) \cdot \mathbf{V}(\mathbf{x})$ has been found, the transmitted field $P_{t}$ follows from an integral over $\mathscr{S}^{10}$

$$
\begin{aligned}
P_{t}(\mathbf{x})= & -\int_{\mathbf{x}^{\prime} \in \mathscr{P}}\left[\Gamma_{2}\left(\mathbf{x} \mid \mathbf{x}^{\prime}\right) \mathbf{V}\left(\mathbf{x}^{\prime}\right)+\boldsymbol{\Lambda}_{2}\left(\mathbf{x} \mid \mathbf{x}^{\prime}\right) P\left(\mathbf{x}^{\prime}\right)\right] \\
& \cdot \boldsymbol{\nu}\left(\mathbf{x}^{\prime}\right) d \mathbf{x}^{\prime}, \quad \mathbf{x} \in \mathscr{D}_{2} .
\end{aligned}
$$

The numerical solution to (1) and (2) can be obtained by discretizing the integral equations, evaluating the singular parts of the integrals, and solving the resulting system of linear equations by matrix inversion. This method will be called the direct integral equation method (DIE).

\section{B. Iterative solution method}

The technique described in Ref. 8 is used to arrive at an iterative method for the solution of the two coupled integral equations. The iterations are obtained from a continuous version of the conjugate gradient technique. The method will be called the conjugate gradient integral equation method (CGIE). Normalizing the unknowns $P(\mathbf{x})$ and $V(\mathbf{x})$ $=\mathbf{V}(\mathbf{x}) \cdot \boldsymbol{\nu}(\mathbf{x})$ according to

$$
\begin{aligned}
& P(\mathbf{x})=Z_{0}^{1 / 2} X_{P}(\mathbf{x}), \\
& V(\mathbf{x})=Y_{0}^{1 / 2} X_{V}(\mathbf{x}),
\end{aligned}
$$

with $Z_{0}$ and $Y_{0}$ a reference impedance and admittance, respectively, the integral equations (1) and (2) can be written as

$$
\begin{aligned}
Y_{1}(\mathbf{x})= & \int_{\mathbf{x}^{\prime} \in \mathscr{S}}\left[K_{1 P}\left(\mathbf{x}, \mathbf{x}^{\prime}\right) X_{P}\left(\mathbf{x}^{\prime}\right)\right. \\
& \left.+K_{1 V}\left(\mathbf{x}, \mathbf{x}^{\prime}\right) X_{V}\left(\mathbf{x}^{\prime}\right)\right] d \mathbf{x}^{\prime}, \quad \mathbf{x} \in \mathscr{S}, \\
Y_{2}(\mathbf{x})= & \int_{\mathbf{x}^{\prime} \in \mathscr{S}}\left[K_{2 P}\left(\mathbf{x}, \mathbf{x}^{\prime}\right) X_{P}\left(\mathbf{x}^{\prime}\right)\right. \\
& \left.+K_{2 V}\left(\mathbf{x}, \mathbf{x}^{\prime}\right) X_{V}\left(\mathbf{x}^{\prime}\right)\right] d \mathbf{x}^{\prime}, \quad \mathbf{x} \in \mathscr{S} .
\end{aligned}
$$

We assume the existence of an iterative procedure, in which $n$ steps have been carried out. The iterative procedure has led 
to the values $X_{P}^{(n)}(\mathbf{x})$ and $X_{V}^{(n)}(\mathbf{x})$. The integrated squared error after $n$ steps of iteration is

$$
\operatorname{ERR}^{(n)}=\int_{\mathbf{x} \in \mathscr{S}}\left(\left|F_{1}^{(n)}(\mathbf{x})\right|^{2}+\left|F_{2}^{(n)}(\mathbf{x})\right|^{2}\right) d \mathbf{x},
$$

in which

$$
\begin{aligned}
F_{1,2}^{(n)}(\mathbf{x})= & Y_{1,2}(\mathbf{x})-\int_{\mathbf{x}^{\prime} \in \mathscr{P}}\left[K_{1,2 P}\left(\mathbf{x}, \mathbf{x}^{\prime}\right) X_{P}^{(n)}\left(\mathbf{x}^{\prime}\right)\right. \\
& \left.+K_{1,2 V}\left(\mathbf{x}, \mathbf{x}^{\prime}\right) X_{V}^{(n)}\left(\mathbf{x}^{\prime}\right)\right] d \mathbf{x}^{\prime}, \quad \mathbf{x} \in \mathscr{S} .
\end{aligned}
$$

In going from the $(n-1)$ st step to the $n$ th, we use

$$
X_{P, V}^{(n)}=X_{P, V}^{(n-1)}+\eta^{(n)} g_{P, V}^{(n)},
$$

where $\eta^{(n)}$ is a variational parameter to minimize $\operatorname{ERR}^{(n)}$ and $g_{P, V}^{(n)}=g_{P, V}^{(n)}(\mathbf{x})$ are the search directions. Using Eq. (10), it can be shown that

$$
F_{1,2}^{(n)}=F_{1,2}^{(n-1)}-\eta^{(n)} f_{1,2}^{(n)},
$$

in which

$$
\begin{aligned}
f_{1,2}^{(n)}(\mathbf{x})= & \int_{\mathbf{x}^{\prime} \in \mathscr{S}}\left[K_{1,2 P}\left(\mathbf{x}, \mathbf{x}^{\prime}\right) X_{P}^{(n)}\left(\mathbf{x}^{\prime}\right)\right. \\
& \left.+K_{1,2 V}\left(\mathbf{x}, \mathbf{x}^{\prime}\right) X_{V}^{(n)}\left(\mathbf{x}^{\prime}\right)\right] d \mathbf{x}^{\prime}, \quad \mathbf{x} \in \mathscr{S} .
\end{aligned}
$$

For the search directions the conjugate gradient directions are taken: ${ }^{13}$

$$
\begin{aligned}
& g_{P, V}^{(n)}=s_{P, V}^{(n-1)}+\frac{A^{(n)}}{A^{(n-1)}} g_{P, V}^{(n-1)}, \quad n \geqslant 2, \\
& g_{P, V}^{(1)}=s_{P, V}^{(0)},
\end{aligned}
$$

where the symbols $s_{P, V}^{(n)}=s_{P, V}^{(n)}(\mathbf{x})$ denote the steepest-descent directions

$$
\begin{aligned}
s_{P, V}^{(n)}(\mathbf{x})= & {\left[\int _ { \mathbf { x } ^ { \prime } \in \mathscr { S } } \left[K_{1 P, V}\left(\mathbf{x}^{\prime}, \mathbf{x}\right) F_{1}^{(n) *}\left(\mathbf{x}^{\prime}\right)\right.\right.} \\
& \left.\left.+K_{2 P, V}\left(\mathbf{x}^{\prime}, \mathbf{x}\right) F_{2}^{(n) *}\left(\mathbf{x}^{\prime}\right)\right] d \mathbf{x}^{\prime}\right]^{*}, \quad \mathbf{x} \in \mathscr{S},
\end{aligned}
$$

(the asterisk denotes complex conjugate) and

$$
A^{(n)}=\int_{\mathbf{x} \in \mathscr{S}}\left(\left|s_{P}^{(n-1)}\right|^{2}+\left|s_{V}^{(n-1)}\right|^{2}\right) d \mathbf{x} .
$$

The variational parameter resulting from the choice for the conjugate gradient directions is given by

$$
\eta^{(n)}=A^{(n)} / B^{(n)},
$$

with

$$
B^{(n)}=\int_{\mathbf{x} \in \mathscr{S}}\left(\left|f_{1}^{(n)}\right|^{2}+\left|f_{2}^{(n)}\right|^{2}\right) d \mathbf{x} .
$$

The error decreases at each iteration step according to

$$
\operatorname{ERR}^{(n)}=\operatorname{ERR}^{(n-1)}-\frac{\left|A^{(n)}\right|^{2}}{B^{(n)}} .
$$

As a result, a decrease of the error is obtained if $A^{(n)} \neq 0$. From Eq. (16) it can be seen that this is the case if $s_{P, V}^{(n-1)} \neq 0$. However, if $s_{P, V}^{(n-1)}$ vanishes, the exact solution is arrived at in the iteration $n-1$. Once the iteration process has been stopped, the transmitted field is found from evaluation of Eq. (3).

\section{ITERATIVE PLANE-WAVE METHOD}

The solution of the reflection/transmission problem can also be found by expanding the field in a Fourier-type integral of plane waves. ${ }^{9}$ This method will be called the conjugate gradient Rayleigh (CGR) method because of its close relationship with the modified Rayleigh method using direct matrix inversion. ${ }^{14}$ The existence of an iterative procedure is assumed in which $n$ steps have been carried out. The iterative procedure has led to the plane-wave components $\phi_{r}^{(n)}$ and $\phi_{t}^{(n)}$ of the reflected and transmitted velocity potentials, respectively. The corresponding field values are

$$
\begin{aligned}
\left\{P_{r}^{(n)}, \mathbf{V}_{r}^{(n)}\right\}= & \iint_{-\infty}^{\infty}\left\{p_{r}^{(n)}, \mathbf{v}_{r}^{(n)}\right\} e^{i \mathbf{k}_{1}^{+} \cdot \mathbf{x}} d k_{x} d k_{y}, \\
& \mathbf{x} \in \mathscr{D}_{1}, \\
\left\{P_{t}^{(n)}, \mathbf{V}_{t}^{(n)}\right\}= & \iint_{-\infty}^{\infty}\left\{p_{t}^{(n)}, \mathbf{v}_{t}^{(n)}\right\} e^{i \mathbf{k}_{2}^{-} \cdot \mathbf{x}} d k_{x} d k_{y}, \\
& \mathbf{x} \in \mathscr{D}_{2},
\end{aligned}
$$

where

$$
\begin{gathered}
\left\{p_{r}^{(n)}, \mathbf{v}_{r}^{(n)}\right\}=i\left\{\omega \rho_{1}, \mathbf{k}_{1}^{+}\right\} \phi_{r}^{(n)}, \\
\left\{p_{t}^{(n)}, \mathbf{v}_{t}^{(n)}\right\}=i\left\{\omega \rho_{2}, \mathbf{k}_{2}^{-}\right\} \phi_{t}^{(n)} .
\end{gathered}
$$

The plane-wave vectors $\mathbf{k}_{1}^{+}$and $\mathbf{k}_{2}^{-}$indicate plane waves traveling away from $\mathscr{S}$ :

$$
\mathbf{k}_{1}^{+}=\left(k_{x}, k_{y}, k_{z, 1}\right),
$$

with

$$
k_{z, 1}=\left(\omega^{2} \rho_{1} \kappa_{1}-k_{x}^{2}-k_{y}^{2}\right)^{1 / 2}, \quad \Re\left(k_{z, 1}\right), \Im\left(k_{z, 1}\right)>0,
$$

and

$$
\mathbf{k}_{2}^{-}=\left(k_{x}, k_{y},-k_{z, 2}\right),
$$

with

$$
k_{z, 2}=\left(\omega^{2} \rho_{2} \kappa_{2}-k_{x}^{2}-k_{y}^{2}\right)^{1 / 2}, \quad \Re\left(k_{z, 2}\right), \Im\left(k_{z, 2}\right)>0 .
$$

The integrated squared error $\operatorname{ERR}^{(n)}$ in the boundary conditions after $n$ steps of iteration is

$$
\operatorname{ERR}^{(n)}=\int_{\mathbf{x} \in \mathscr{S}}\left(\left|F_{P}^{(n)}\right|^{2}+\left|F_{V}^{(n)}\right|^{2}\right) d \mathbf{x},
$$

in which the deviations $F_{P}^{(n)}=F_{P}^{(n)}(\mathbf{x}), F_{V}^{(n)}=F_{V}^{(n)}(\mathbf{x})$ are given by

$$
\begin{aligned}
& F_{P}^{(n)}=Y_{0}^{1 / 2}\left(P_{i}+P_{r}^{(n)}-P_{t}^{(n)}\right), \\
& F_{V}^{(n)}=Z_{0}^{1 / 2} \boldsymbol{\nu} \cdot\left(\mathbf{V}_{i}+\mathbf{V}_{r}^{(n)}-\mathbf{V}_{t}^{(n)}\right) .
\end{aligned}
$$

In going from the $(n-1)$ st step to the $n$ th, we use

$$
\phi_{r, t}^{(n)}=\phi_{r, t}^{(n-1)}+\eta^{(n)} g_{r, t}^{(n)},
$$


where $\eta^{(n)}$ is a variational parameter to minimize $\operatorname{ERR}^{(n)}$ and $g_{r, t}^{(n)}=g_{r, t}^{(n)}\left(k_{x}, k_{y}\right)$ are the search directions. Upon using Eq. (29) it can be shown that

$$
F_{P, V}^{(n)}=F_{P, V}^{(n-1)}-\eta^{(n)} f_{P, V}^{(n)},
$$

where

$$
\begin{aligned}
f_{P}^{(n)}= & -i \omega \rho_{1} Y_{0}^{1 / 2} \iint_{-\infty}^{\infty} g_{r}^{(n)} e^{i \mathbf{k}_{1}^{+} \cdot \mathbf{x}} d k_{x} d k_{y} \\
& +i \omega \rho_{2} Y_{0}^{1 / 2} \iint_{-\infty}^{\infty} g_{t}^{(n)} e^{i \mathbf{k}_{2}^{-} \cdot \mathbf{x}} d k_{x} d k_{y}, \\
f_{V}^{(n)}= & -i Z_{0}^{1 / 2} \iint_{-\infty}^{\infty} \boldsymbol{\nu} \cdot \mathbf{k}_{1}^{+} g_{r}^{(n)} e^{i \mathbf{k}_{1}^{+} \cdot \mathbf{x}} d k_{x} d k_{y} \\
& +i Z_{0}^{1 / 2} \iint_{-\infty}^{\infty} \boldsymbol{\nu} \cdot \mathbf{k}_{2}^{-} g_{t}^{(n)} e^{i \mathbf{k}_{2}^{-} \cdot \mathbf{x}} d k_{x} d k_{y} .
\end{aligned}
$$

For the search directions the conjugate gradient directions are taken

$$
\begin{aligned}
& g_{r, t}^{(n)}=s_{r, t}^{(n-1)}+\frac{A^{(n)}}{A^{(n-1)}} g_{r, t}^{(n-1)}, \quad n \geqslant 2, \\
& g_{r, t}^{(1)}=s_{r, t}^{(0)} .
\end{aligned}
$$

where the symbols $s_{r, t}^{(n)}=s_{r, t}^{(n)}\left(k_{x}, k_{y}\right)$ represent the steepestdescent directions

$$
\begin{aligned}
s_{r}^{(n)}= & {\left[-i \omega \rho_{1} Y_{0}^{1 / 2} \int_{\mathbf{x} \in \mathscr{S}} F_{P}^{(n) *} e^{i \mathbf{k}_{1}^{+} \cdot \mathbf{x}} d \mathbf{x}\right.} \\
& \left.-i Z_{0}^{1 / 2} \int_{\mathbf{x} \in \mathscr{S}} \boldsymbol{\nu} \cdot \mathbf{k}_{1}^{+} F_{V}^{(n) *} e^{i \mathbf{k}_{1}^{+} \cdot \mathbf{x}} d \mathbf{x}\right]^{*}, \\
s_{t}^{(n)}= & {\left[+i \omega \rho_{2} Y_{0}^{1 / 2} \int_{\mathbf{x} \in \mathscr{S}} F_{P}^{(n) *} e^{i \mathbf{k}_{2}^{-} \cdot \mathbf{x}} d \mathbf{x}\right.} \\
& \left.+i Z_{0}^{1 / 2} \int_{\mathbf{x} \in \mathscr{S}} \boldsymbol{\nu} \cdot \mathbf{k}_{2}^{-} F_{V}^{(n) *} e^{i \mathbf{k}_{2}^{-} \cdot \mathbf{x}} d \mathbf{x}\right]^{*},
\end{aligned}
$$

and

$$
A^{(n)}=\iint_{-\infty}^{\infty}\left(\left|s_{r}^{(n-1)}\right|^{2}+\left|s_{t}^{(n-1)}\right|^{2}\right) d k_{x} d k_{y} .
$$

The variational parameter is given by $\eta^{(n)}=A^{(n)} / B^{(n)}$ where

$$
B^{(n)}=\int_{\mathbf{x} \in \mathscr{S}}\left(\left|f_{P}^{(n)}\right|^{2}+\left|f_{V}^{(n)}\right|^{2}\right) d \mathbf{x} .
$$

Again a decrease of the error is obtained: $\mathrm{ERR}^{(n)}<\mathrm{ERR}^{(n-1)}$. The transmitted field follows from evaluation of Eq. (21).

\section{NUMERICAL IMPLEMENTATION}

\section{A. Weighting functions}

Some issues concerning the 2-D implementation are presented. To be able to make a fair comparison between the integral equation methods and the plane-wave method, the same spatial weighting function was used. For the comparison described in this paper, Dirac-impulse functions were used. This results in the so-called point-matching technique, where the integrations are performed as simple summations of the discrete function values. If piecewise polynomials are used for both the weighting functions and the description of the surface, then the subintegrals of the CGR method can be evaluated analytically. The CGR scheme was implemented for two different weighting functions: the Dirac-impulse function and the piecewise constant function. The implementation for different weighting functions also provides a convenient check for the required spatial integration step size. If the root-mean-square difference in the solution for Diracweighting and piecewise constant weighting was larger than, say, one percent, then the step size was rejected as being too large.

\section{B. Integrals of Green's functions}

The Hankel functions of the integral equation methods were calculated by using the polynomial descriptions in (Ref. 15, pp. 369-370). Although the latter expressions are intended for real-valued arguments, complex arguments with relatively small imaginary parts also may be used. For the absorption typically found in soft human tissue, these expressions are accurate to about seven significant digits, where recursion relations (Ref. 15, pp. 385-386) were used as reference. When applying the Dirac-weighting functions, exceptions have to be made for the integrable singularities of the integral equation kernels, which were evaluated by using a piecewise constant weighting function. These singular parts of the integrals were evaluated analytically. ${ }^{16}$ The subintegral of the singular derivative of the Hankel function was assumed to be zero.

\section{Spatial discretization}

Experiments with the spatial step size showed that, for small to moderate surface slopes, the calculations gave consistent results for a spatial integration step size $\Delta x \leqslant 0.2 \lambda$, where $\lambda$ is the smallest wavelength in both media. For larger surface slopes the discretization in the $x$ direction should be smaller. As an alternative, the discretization can be carried out with constant step size along the surface. To study the behavior of the methods for varying surface characteristics, surfaces with different correlation lengths $L$ and different root-mean-square surface heights $h$ were generated. The irregular interface has a surface height with a Gaussian roughness spectrum, as described by Thorsos. ${ }^{16}$ A surface realization with correlation length $L=1 \mathrm{~mm}$ and rms surface height $h=0.5 \mathrm{~mm}$ is shown in Fig. 1. The convergence of the CGR method is primarily governed by the maximum surface slope. ${ }^{9}$ Therefore the ratio $h / L$ was used as one of the independent parameters. The other independent parameter was the correlation length $L$. For $h / L \leqslant 0.5,512$ surface points at a step size $\Delta x=0.2 \lambda$ were used, with $\lambda=2 \pi /\left(\omega \sqrt{\rho_{2} \kappa_{2}}\right)$ the wavelength in medium 2 . For $h / L=1,1024$ surface points with $\Delta x=0.1 \lambda$ were used.

\section{Spectral discretization}

The CGIE method uses the same function spaces for the residuals as for the search directions. For the CGR method 


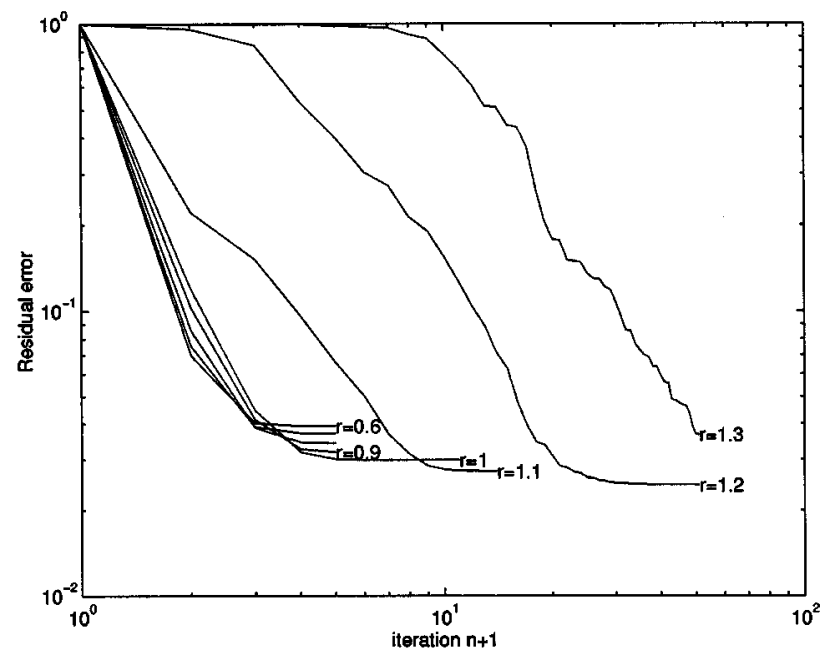

FIG. 2. Residual error $\operatorname{ERR}^{(n)}$ of the CGR method as a function of the iteration number $n$ with parameter the ratio $r$, the total number of plane waves taken into account divided by the number of propagating waves; $L=\lambda=0.294 \mathrm{~mm}, h=\lambda / 2=0.147 \mathrm{~mm}$.

however, residuals are calculated in the spatial domain, whereas, the search directions are calculated in the spectral domain. This leads to an additional degree of freedom with respect to the discrete implementation. However, it will be seen that the discretization in the spatial domain prescribes the choice for the (optimum) discretization in the spectral domain. This is illustrated in Fig. 2, where the convergence properties of the CGR method are shown. The plot shows the residual error as a function of the number of iterations. The residual error $\widehat{E R R}^{(\mathrm{n})}$ at iteration $n$ is defined by

$$
\operatorname{ERR}^{(n)}=\left(\frac{\operatorname{ERR}^{(n)}}{\mathrm{ERR}_{0}}\right)^{1 / 2},
$$

where $\mathrm{ERR}_{0}$ is the initial error $\mathrm{ERR}^{(0)}$ for a zero initial guess, i.e., $X_{P, V}^{(0)}=0$ (CGIE) or $\phi_{r, t}^{(0)}=0$ (CGR). The ratio $r$ is introduced as the total number of modes taken into account divided by the number of propagating modes. In fact, the graph of Fig. 2 shows the convergence properties for a fixed spectral step size with the number of modes as parameter. It can be seen that the error reaches a certain minimum value after some iteration steps, where the minimum is lower if more modes are included. However, it can also be seen that the convergence rate strongly decreases if $r$ becomes larger than unity, i.e., if more evanescent modes are included. In the next subsection it will be shown how a preconditioning operator can improve the convergence considerably for this case. However, in almost every case occurring in our application, we found that the use of propagating modes only, leads to a sufficiently small residual error. If we omit the evanescent modes altogether, the question remains what the desired number of propagating modes is. To this end, all modes up to the evanescent mode limit were used where the mesh size in the discretized spectral domain was varied. This is equivalent to varying the number of propagating modes, which is shown in Fig. 3. The dashed line indicates the number of propagating modes corresponding to the discrete Fourier transform, for a given spatial stepsize/wavelength ratio. Figure 3 shows that the residual error is not improved any-

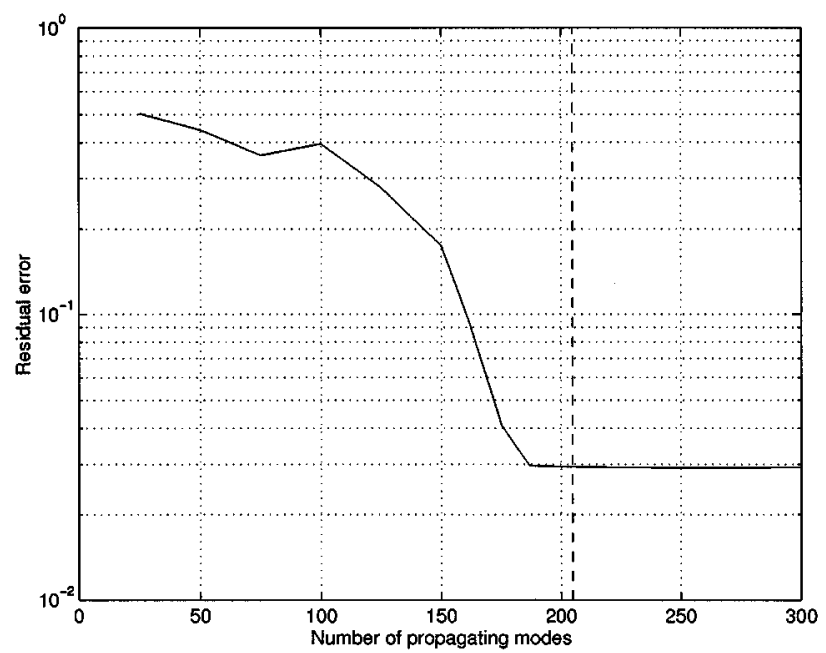

FIG. 3. Final residual error $\widehat{E R R}^{(N)}$ of the CGR method as a function of the number of propagating modes. Evanescent modes are omitted. The dashed line corresponds to the number of propagating modes for the discrete Fourier transform: $2 N_{x} \Delta x / \lambda$, with $N_{x}=512, \Delta x / \lambda=0.2, L=1 \mathrm{~mm}, h=0.5 \mathrm{~mm}$.

more if the number of modes is larger than the number of modes for the discrete Fourier transform. Taking the spectral stepsize corresponding to the discrete Fourier transform turns out to be very convenient in practice. It allows a direct coupling of the rough surface calculation method to FFT-based extrapolation methods for plano-parallel geometries. Following the above, the number of plane waves for the CGR method was taken to be equal to the number of surface points $N_{x}$. The spectral step size $\Delta k_{x}$ was taken to be the value for the discrete Fourier transform, i.e., $\Delta k_{x}=2 \pi /\left(N_{x} \Delta x\right)$. The number of unknowns then becomes $2 N_{x}$ if all modes are used and $4 N_{x} \Delta x / \lambda$ if only the propagating modes are used.

\section{E. Preconditioning}

In the previous subsection, the inadequate convergence of the CGR method was mentioned in the case that evanescent modes were included. If the problem would have been solved with direct matrix inversion, then the matrix would have been found to be very ill conditioned. ${ }^{17}$ Ill-conditioned matrices often lead to badly converging conjugate gradient schemes. ${ }^{18}$ Therefore, a modification of the scheme was searched for by employing well-behaved matrix elements. The modification was implemented along the lines of Ref. 18 by using a preconditioning operator $P$ that approximates the inverse operator of the problem at hand. The conjugate gradient directions for the preconditioned scheme become

$$
\begin{aligned}
& g_{r, t}^{(n)}=P^{*} P s_{r, t}^{(n-1)}+\frac{A^{(n)}}{A^{(n-1)}} g_{r, t}^{(n-1)}, \quad n \geqslant 2, \\
& g_{r, t}^{(1)}=P^{*} P s_{r, t}^{(0)},
\end{aligned}
$$

where $P^{*}$ is the adjoint of $P$ and where

$$
A^{(n)}=\iint_{-\infty}^{\infty}\left(\left|P s_{r}^{(n-1)}\right|^{2}+\left|P s_{t}^{(n-1)}\right|^{2}\right) d k_{x} d k_{y} .
$$

Various preconditioners were evaluated. A preconditioner which gave useful results is the inverse of the diagonal from 


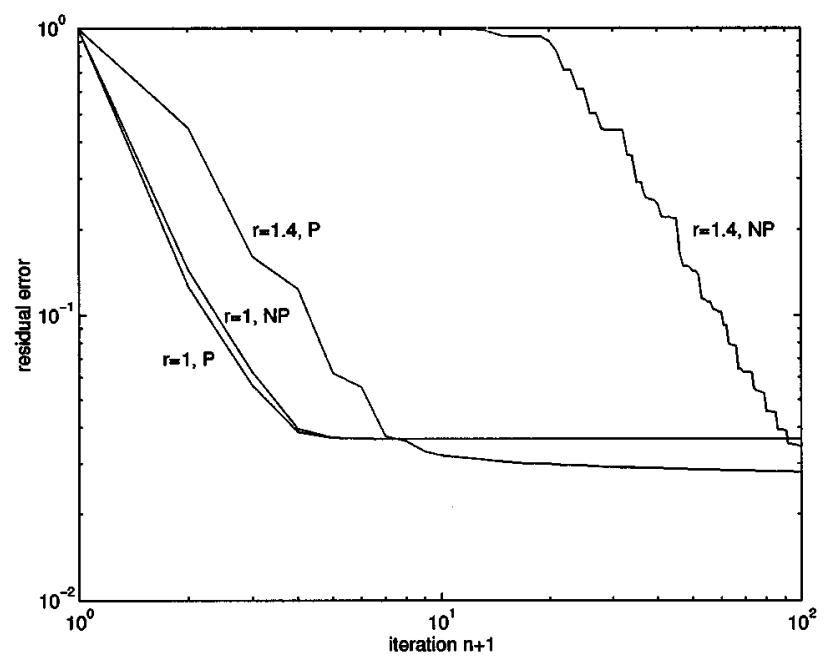

FIG. 4. Residual error $\widehat{E R R}^{(n)}$ of the CGR method with preconditioning (indicated by $\mathrm{P}$ ) and no preconditioning (indicated by NP) as a function of the iteration number $n$, with parameter the ratio $r$, the total number of plane waves taken into account divided by the number of propagating waves; $L=\lambda=0.294 \mathrm{~mm}, h=\lambda / 2=0.147 \mathrm{~mm}, N_{x}=256$.

the direct matrix inversion method of our least-square minimization procedure. Then, the preconditioners are defined by

$$
\begin{aligned}
P s_{r}^{(n)}= & {\left[\int _ { \mathbf { x } \in \mathscr { S } } \left(\left|Y_{0}\right|\left|\omega \rho_{1}\right|^{2}\right.\right.} \\
& \left.\left.+\left|Z_{0}\right|\left|\boldsymbol{\nu} \cdot \mathbf{k}_{1}^{+}\right|^{2}\right)\left|e^{i \mathbf{k}_{1}^{+} \cdot \mathbf{x}}\right|^{2} d \mathbf{x}\right]_{r}^{-1 / 2} s_{r}^{(n)}, \\
P s_{t}^{(n)}= & {\left[\int _ { \mathbf { x } \in \mathscr { S } } \left(\left|Y_{0}\right|\left|\omega \rho_{2}\right|^{2}\right.\right.} \\
& \left.\left.+\left|Z_{0}\right|\left|\boldsymbol{\nu} \cdot \mathbf{k}_{2}^{-}\right|^{2}\right)\left|e^{i \mathbf{k}_{2}^{-} \cdot \mathbf{x}}\right|^{2} d \mathbf{x}\right]^{-1 / 2} s_{t}^{(n)} .
\end{aligned}
$$

In Fig. 4, the convergence of the preconditioned scheme is compared to the convergence of the standard conjugate gradient scheme. From this figure it can be seen that the preconditioning indeed has a positive effect on the convergence rate. It can also be seen, however, that there is little difference in convergence if evanescent modes are omitted. Schemes which are already well behaved, such as the CGR method with omitted evanescent modes, apparently are not substantially improved by using the preconditioner. However, the very ill-conditioned CGR method with evanescent modes benefits from the preconditioner.

\section{RESULTS}

\section{A. Configuration}

The methods were applied to surfaces described by Gaussian statistical averages, in this case by a correlation length $L$ and a root-mean-square surface height $h$. The mean of the surface is at $z=0$. The rough surface is irradiated by an array transducer at depth $z=10 \mathrm{~mm}$. The mean of the interface is at $z=0$. The 1-D linear-array transducer consists of 128 radiating elements, each having a width of $0.15 \mathrm{~mm}$, positioned at a grid distance ("pitch") of $0.2 \mathrm{~mm}$. The elec-

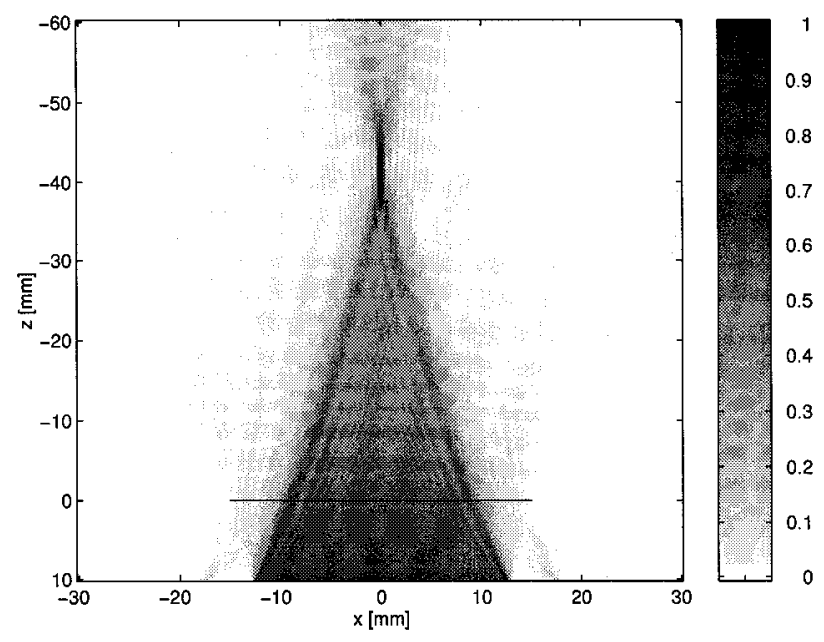

FIG. 5. Pressure magnitude field of array transducer for a plane interface separating two media. The array transducer is positioned at $z=10 \mathrm{~mm}$ and radiates into a medium with parameters $c_{1}=1550 \mathrm{~m} / \mathrm{s}, \rho_{1}=10^{3} \mathrm{~kg} / \mathrm{m}^{3}$ at a frequency of $5 \mathrm{MHz}$. The parameters of the second medium are $c_{2}=1473$ $\mathrm{m} / \mathrm{s}, \rho_{2}=10^{3} \mathrm{~kg} / \mathrm{m}^{3}$. The attenuation in both media is $0.5 \mathrm{~dB} /(\mathrm{cm} \mathrm{MHz})$. The focus is at $z=-40 \mathrm{~mm}$.

tronic focus is at depth $z=-40 \mathrm{~mm}$ (that is, at a distance of $50 \mathrm{~mm}$ from the array), in a medium with the parameters $\rho_{1}$ and $\kappa_{1}$. The array elements radiate with equal magnitudes of normal surface velocity. The frequency is $5 \mathrm{MHz}$ and the sound speeds are $c_{1}=1550 \mathrm{~m} / \mathrm{s}$ and $c_{2}=1473 \mathrm{~m} / \mathrm{s}$, i.e., a sound speed contrast of $5 \%$. The densities are $\rho_{1}=\rho_{2}=10^{3}$ $\mathrm{kg} / \mathrm{m}^{3}$. The attenuation is $0.5 \mathrm{~dB} /(\mathrm{cm} \mathrm{MHz})$. Note that due to the attenuation the compressibilities are complex valued. The values for the reference admittance $Y_{0}$ and the reference impedance $Z_{0}$ were taken to be the geometric means of the admittances and impedances of both media.

\section{B. Performance}

The sound propagation through the irregular interface was analyzed with the two iterative methods and a phasescreen approximation, where the results of the direct integral equation method (DIE) were used as a reference. The eva-

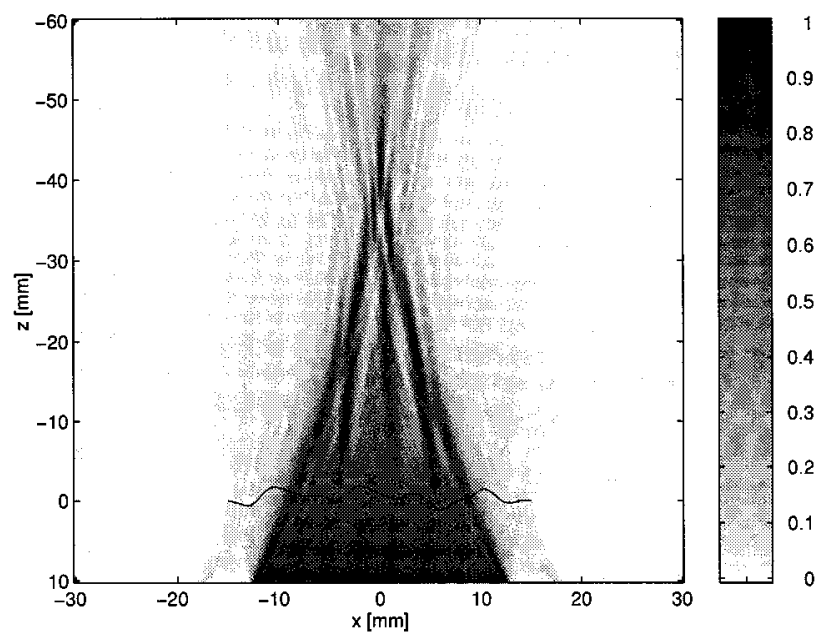

FIG. 6. Pressure magnitude field of array transducer for an irregular interface separating two media with $L=2 \mathrm{~mm}, h=1 \mathrm{~mm}$, CGIE method. 


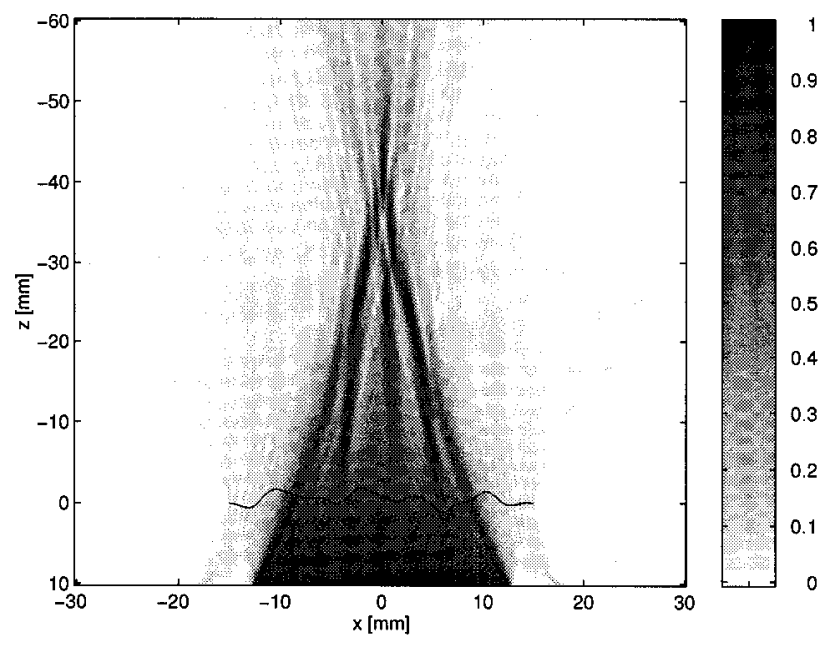

FIG. 7. Pressure magnitude field of array transducer for an irregular interface separating two media with $L=2 \mathrm{~mm}, h=1 \mathrm{~mm}$, CGR method.

nescent components of the CGR method were not taken into account, which results in a relatively high efficiency from a computational point of view. The calculations were run on a 40-MHz Sun-Sparc LX in Matlab 4.2. Within a finite number of iterations the CGIE method always converged to the results obtained with the DIE method. Therefore the CGIE method will be used as the reference from now on.

A beam pressure plot for the plane interface is shown in Fig. 5. The beam pressure plots show the incident pressure field $P_{i}(x)$ and the transmitted pressure field $P_{t}(\mathbf{x})$. The interface is shown as a dark line in the figures. The beam pressure plots for the irregular interface obtained with CGIE (Fig. 6) and CGR (Fig. 7) agree closely.

In Tables I-IV the results are shown for the different surface characteristics. It can be seen that the residual error $\operatorname{ERR}_{\text {CGR }}^{(N)}$ of the CGR method becomes larger for larger $h / L$ ratios, as was also shown in Ref. 9 for sinusoidal interfaces. On the surface, the normalized root-mean-square difference $E_{\text {CGIE-CGR }}$ between the CGR method and the integral equation methods has a similar behavior. The CGR residual is roughly a factor 2 smaller than the difference between the two methods. If we assume that the integral equation gives the more accurate result then we can say that the residual of the CGR method is a good indication of the absolute accuracy on the surface. The performance in the focal plane has a somewhat different behavior. At first sight, the difference $E_{\text {CGIE-CGR }}$ between the CGR method and the integral equation methods does not seem to depend on the surface irregularity for small surface irregularities. As a matter of fact, the rms difference for a completely flat interface is 0.054 . This difference is caused by the finite support of the spatial integrals. At a distance halfway the focal plane, the differences between the iterative methods are smaller. In Tables I-IV also the results for a phase-screen approximation are shown. The tables show the normalized root-mean-square difference $E_{\text {CGIE-phases screen }}$ between the phase-screen method and the integral equation methods. The phase-screen approximation acts as a local time-shift operator based on travel time differences in the two media. Refraction is not taken into account in the phase-screen approximation, which leads to rela-
TABLE I. Residual error $\operatorname{ERR}_{\text {CGR }}^{(N)}$ of the CGR method, the normalized rootmean-square difference between the integral equation methods and the CGR method $E_{\text {CGIE-CGR }}$ (on the rough surface) and $E_{\text {CGIE-CGR }}$ (in the focal plane), and the normalized root-mean-square difference between the integral equation methods and the phase-screen method $E_{\text {CGIE-phase screen }}$, for $h / L=0.1$.

\begin{tabular}{ccccc}
\hline \hline$L[\mathrm{~mm}]$ & $\widehat{E R R}_{\text {CGR }}^{(N)}$ & $\begin{array}{c}E_{\text {CGIE-CGR }} \\
\text { (rough interface) }\end{array}$ & $\begin{array}{c}E_{\text {CGIE-CGR }} \\
\text { (focal plane) }\end{array}$ & $\begin{array}{c}E_{\text {CGIE-phase screen }} \\
\text { (focal plane) }\end{array}$ \\
\hline 0.2 & 0.0040 & 0.0090 & 0.048 & 0.062 \\
0.5 & 0.0013 & 0.0042 & 0.048 & 0.061 \\
1 & 0.0010 & 0.0030 & 0.047 & 0.060 \\
2 & 0.0024 & 0.0055 & 0.047 & 0.061 \\
\hline \hline
\end{tabular}

TABLE II. As Table I, except $h / L=0.2$.

\begin{tabular}{lcccc}
\hline \hline$L[\mathrm{~mm}]$ & $\operatorname{ERR}_{\mathrm{CGR}}^{(N)}$ & $\begin{array}{c}E_{\text {CGIE-CGR }} \\
\text { (rough interface) }\end{array}$ & $\begin{array}{c}E_{\text {CGIE-CGR }} \\
\text { (focal plane) }\end{array}$ & $\begin{array}{c}E_{\text {CGIE-phase screen }} \\
\text { (focal plane) }\end{array}$ \\
\hline 0.2 & 0.0089 & 0.022 & 0.049 & 0.065 \\
0.5 & 0.0048 & 0.012 & 0.047 & 0.060 \\
1 & 0.0095 & 0.018 & 0.047 & 0.062 \\
2 & 0.0049 & 0.0086 & 0.046 & 0.063 \\
\hline \hline
\end{tabular}

TABLE III. As Table I, except $h / L=0.5$.

\begin{tabular}{lcccc}
\hline \hline$L[\mathrm{~mm}]$ & $\operatorname{ERR}_{\mathrm{CGR}}^{(N)}$ & $\begin{array}{c}E_{\text {CGIE-CGR }} \\
\text { (rough interface) }\end{array}$ & $\begin{array}{c}E_{\text {CGIE-CGR }} \\
\text { (focal plane) }\end{array}$ & $\begin{array}{c}E_{\text {CGIE-phase screen }} \\
\text { (focal plane) }\end{array}$ \\
\hline 0.2 & 0.036 & 0.070 & 0.065 & 0.092 \\
0.5 & 0.035 & 0.065 & 0.050 & 0.071 \\
1 & 0.040 & 0.080 & 0.047 & 0.078 \\
2 & 0.051 & 0.085 & 0.047 & 0.12 \\
\hline \hline
\end{tabular}

TABLE IV. As Table I, except $h / L=1$.

\begin{tabular}{lcccc}
\hline \hline$L[\mathrm{~mm}]$ & $\operatorname{ERR}_{\text {CGR }}^{(N)}$ & $\begin{array}{c}E_{\text {CGIE-CGR }} \\
\text { (rough interface) }\end{array}$ & $\begin{array}{c}E_{\text {CGIE-CGR }} \\
\text { (focal plane) }\end{array}$ & $\begin{array}{c}E_{\text {CGIE-phase screen }} \\
\text { (focal plane) }\end{array}$ \\
\hline 0.2 & 0.090 & 0.13 & 0.078 & 0.13 \\
0.5 & 0.086 & 0.17 & 0.070 & 0.14 \\
1 & 0.076 & 0.22 & 0.092 & 0.15 \\
2 & 0.058 & 0.16 & 0.072 & 0.39 \\
\hline \hline
\end{tabular}

tively large errors at large $h / L$ ratios. The beam magnitude cross sections for the iterative methods are very close, also in the focal plane. Examples of two beam cross-sections in the focal plane are shown in Figs. 8 and 9. Figure 8 shows the beam cross section for a perfectly flat interface, whereas Fig. 9 shows the beam cross section for a rough interface. It should be noted that the CGR method can be made more accurate than the results of Tables I-IV by using all propagating and evanescent waves, in combination with the preconditioned scheme.

As far as the computational efficiency can be evaluated from a single numerical implementation, it can be said that for the accuracy we desire, the total computation time of the CGIE method is much larger than the computation time of 


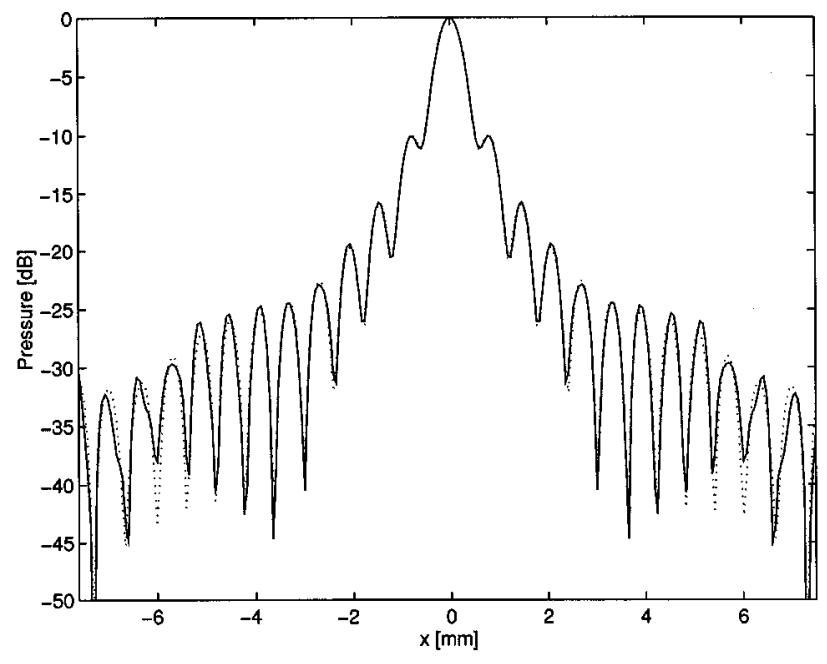

FIG. 8. Magnitude beam cross section of the pressure field in the focus of a linear array transducer for the CGIE method (solid line) and the CGR method (dotted line); plane surface.

CGR (Fig. 10). There are two reasons for this. First, for the first few iteration steps, the convergence of CGIE is not as fast as the convergence of CGR. Second, the computation of the Hankel functions for CGIE requires much more time than the computation of the exponentials in CGR. However, the error obtained with the CGR method reaches a steady value after a number of iterations, whereas the error obtained with CGIE can be driven to an arbitrarily small value. Our interest is in simulation of interfaces of moderate surface slopes separating media with low contrast. Then, the final error value which can be obtained with CGR is sufficiently small.

\section{CONCLUDING REMARKS}

Two iterative methods for the 2-D simulation of wave propagation through aberrating interfaces were compared. The iterative plane-wave (CGR) method has a number of attractive features that makes it very suitable for simulation through aberrating media in human tissue. A clear advantage

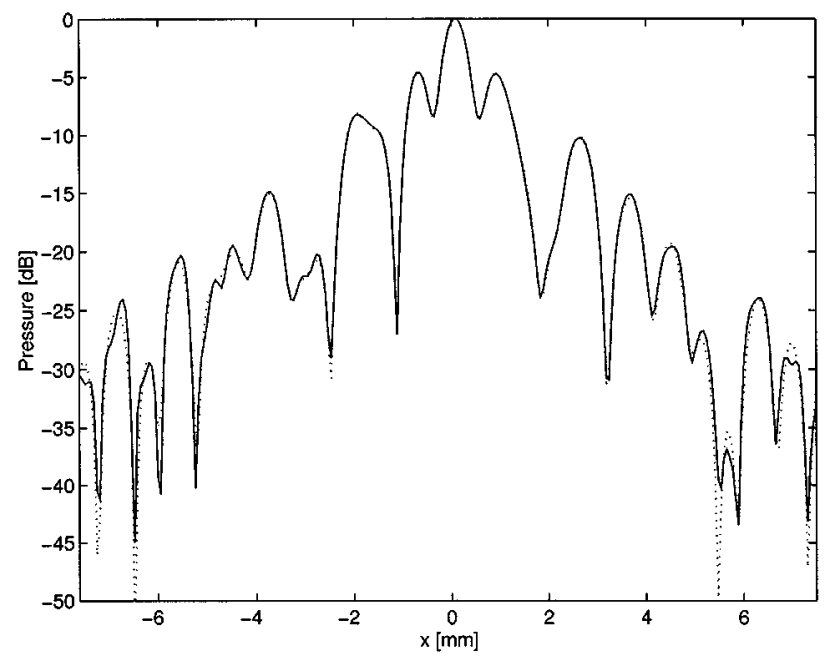

FIG. 9. Magnitude beam cross section of the pressure field in the focus of a linear array transducer for the CGIE method (solid line) and the CGR method (dotted line); surface with Gaussian roughness spectrum having parameters $L=2 \mathrm{~mm}, h=1 \mathrm{~mm}$.

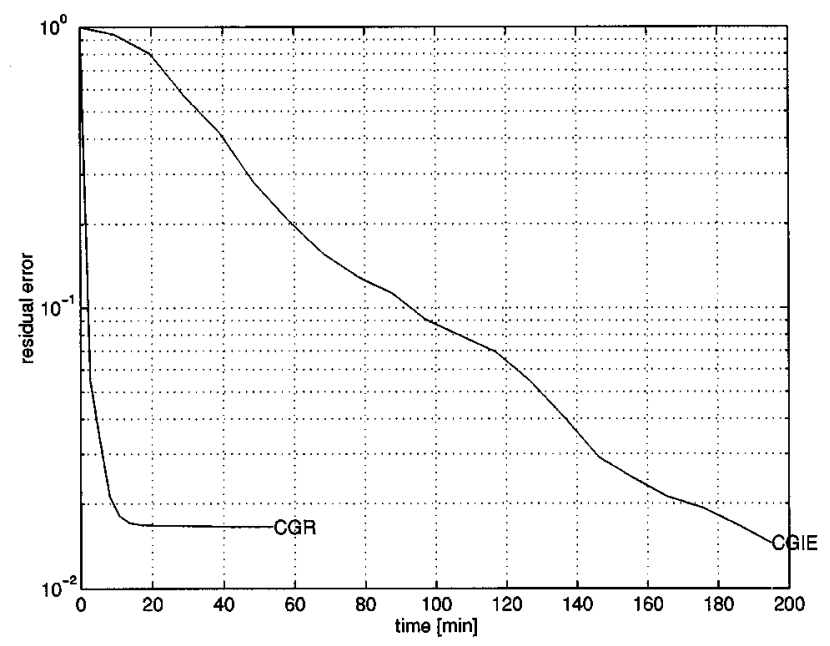

FIG. 10. Residual error $\widehat{\operatorname{ERR}}^{(n)}$ versus computation time on a 40-MHz SunSparc for the CGIE method and the CGR method.

is the very rapid convergence for media with relatively low contrast and moderate surface roughness. The advantage is for a large part obtained from the observation that evanescent modes can safely be neglected. Also in the latter case, the method is more accurate than a phase-screen approximation. For the cases that evanescent modes are required, an efficient preconditioning scheme has been presented. A further advantage of the CGR method is that it can be directly coupled to FFT-based extrapolation methods. In addition, the implementation is relatively simple (also for 3D) because of the lack of singular integrands. For improved accuracy and stability the spatial subintegrals can be evaluated analytically for a common class of surfaces and weighting functions. An often overlooked advantage of plane-wave methods over Green's function methods is that the algorithms can remain unchanged for strongly absorptive media. For this condition, the Hankel functions of the integral equation methods should be calculated in a more sophisticated way.

However, if extremely rough surfaces have to be evaluated, possibly with reentrant points, then the integral equation method should be used.

\section{ACKNOWLEDGMENTS}

This work has been supported by a grant from Philips Medical Systems B.V., Best, The Netherlands and by a grant from the Technological Science Branch of the Dutch Organization for Scientific Research, project NGN 11.2427.

${ }^{1}$ L. Nock, G. E. Trahey, and S. W. Smith, "Phase aberration correction in medical ultrasound using speckle brightness as a quality factor," J. Acoust. Soc. Am. 85, 1819-1833 (1989).

${ }^{2}$ C. Gambetti and F. S. Foster, "Correction of phase aberrations for sectored annular array ultrasound transducers," Ultrasound Med. Biol. 19, 763-776 (1993).

${ }^{3}$ M. Karaman, A. Atalar, H. Kömen, and M. O'Donnell, "A phase aberration correction method for ultrasound imaging," IEEE Trans. Ultrason. Ferroelec. Freq. Control, 40, 275-282 (1993).

${ }^{4}$ D. L. Liu and R. C. Waag, "Time-shift compensation of ultrasonic pulse focus degradation using least-mean-square error estimates of arrival time," J. Acoust. Soc. Am. 95, 542-555 (1994).

${ }^{5} \mathrm{Q}$. Zhu and B. D. Steinberg, "Modeling, measurement and correction of wavefront distortion produced by breast specimens," Proceedings of the 
Ultrasonics Symposium (IEEE, New York, 1994), pp. 1613-1617.

${ }^{6}$ B. S. Robinson, A. Shmulewitz, and T. M. Burke, "Waveform aberrations in an animal model," Proceedings of the Ultrasonics Symposium (IEEE, New York, 1994), pp. 1619-1624.

${ }^{7}$ Q. Zhu and B. D. Steinberg, "Wavefront amplitude distribution in the female breast," J. Acoust. Soc. Am. 96, 1-9 (1994).

${ }^{8} \mathrm{P}$. M. van den Berg, "Iterative computational techniques in scattering based upon the integrated square error criterion," IEEE Trans. Antennas Propag. AP-32, 1063-1071 (1984).

${ }^{9}$ A. P. Berkhoff, P. M. van den Berg, and J. M. Thijssen, "Iterative calculation of reflected and transmitted acoustic waves at a rough interface," IEEE Trans. Ultrason. Ferroelec. Freq. Control 42, 663-671 (1995).

${ }^{10}$ J. T. Fokkema and P. M. van den Berg, Seismic Applications of Acoustic Reciprocity (Elsevier, Amsterdam, 1993).

${ }^{11}$ J. A. Ogilvy, Theory of Wave Scattering from Random Rough Surfaces (IOP, London, 1991).

${ }^{12}$ P. Altmeyer, S. el-Gammal, and K. Hoffmann, Ultrasound in Dermatology
(Springer-Verlag, Berlin, 1992).

${ }^{13}$ P. M. van den Berg, "Iterative schemes based on minimization of a uniform error criterion," in Application of Conjugate Gradient Method to Electromagnetics and Signal Analysis, PIER 5, edited by T. K. Sarkar (Elsevier, Amsterdam, 1991), Chap. 2, pp. 27-65.

${ }^{14}$ P. M. van den Berg, "Reflection by a grating: Rayleigh methods," J. Opt. Soc. Am. 10, 1224-1229 (1981).

${ }^{15}$ M. Abramowitz and I. A. Stegun, Handbook of Mathematical Functions (Dover, New York, 1972).

${ }^{16}$ E. I. Thorsos, "The validity of the Kirchhoff approximation for rough surface scattering using a Gaussian roughness spectrum," J. Acoust. Soc. Am. 83, 78-92 (1988).

${ }^{17} \mathrm{~J}$-M. Chesneaux and A. Wirgin, "Reflection from a corrugated surface revisited,” J. Acoust. Soc. Am. 96, 1116-1129 (1994).

${ }^{18}$ G. H. Golub and C. F. van Loan, Matrix Computations (John Hopkins U.P., Baltimore, 1989), pp. 527-529. 